This is a peer-reviewed, accepted author manuscript of the following article: Yuan, M., Wang, J., Wang, M., Huang, K., Wang, L., \& Tian, Y. (2020). Enhanced carbide tool life by the electromagnetic coupling field for sustainable manufacturing. International Journal of Advanced Manufacturing Technology , 108(11-12), 3905-3914. https://doi.org/10.1007/s00170-020-05612-3

\title{
Enhanced carbide tool life by the electromagnetic coupling field for sustainable manufacturing
}

\author{
Yuan Min ${ }^{1}$, Wang Jie ${ }^{1, *}$, Wang Mei ${ }^{1}$, Huang Kunlan ${ }^{1}$, Wang $\mathrm{Li}^{1}$, Yankang Tian ${ }^{2}$
}

1School of Mechanical Engineering, Sichuan University, Chengdu, Sichuan 610065, P.R. China

2Centre for Precision Manufacturing and Micro-Manufacturing, Dept. of DMEM, University of Strathclyde, James

Weir Building, 75 Montrose Street, Glasgow G1 1XJ, UK

* Corresponding author: E-mail address: wangjie @scu.edu.cn

\begin{abstract}
The effective extension of tool life while maintaining machining quality is an important research topic in advanced machining and sustainable manufacturing. Cemented carbide is widely used as the tool material in different manufacturing processes, and it has various forms and work ranges. However, the internal flaw in the tool material can induce a micro crack which could result in the decrease of tool strength and toughness and affect the tool life. Improving the tool cutting performance, slowing down the tool wear, and enhancing production efficiency are the eternal themes of cutting tool research. This research focused on a P10 cemented carbide tool. The influences of the electromagnetic coupling field (TEMCP) on the carbide tool life and the maximum of tool force are investigated. The correlation analysis between the TEMCP parameters and the tool life index is conducted using SPSS. The experiment proves that the TEMCP can significantly prolong the cemented carbide tool life, and that the magnetic intensity is a dominant factor. The TEMCP enriches the field technology theory and provides technical support for the sustainable manufacturing and research and development of a high-performance tool with important scientific meaning and research potential.
\end{abstract}

Keywords: electromagnetic coupling field; tool life; carbide tool; force signal

\section{Introduction}

The modern machining technology is developing toward the direction of high speed, high precision, and high-rate cutting. The performance, quality, and reliability of the cutting tool material directly affect the whole mechanical manufacturing industry's production technology level and economic benefits [1]. Traditional materials and their forming technologies cannot satisfy the growing demand of the high-end application market because of the increasing speed of product upgrades. Many studies on cutting tools have been conducted around the maximum tool cutting performance, reducing tool wear, extending tool life, and improving the quality of processed workpiece surfaces [2].On the contrary, the development of new technologies is no longer a simple technology improvement, but already a reflection of innovation. Further requirements about environmental protection and energy saving have been presented [3].

Fully exploring the tool cutting performance, slowing down the tool wear, and improving the 
ability of cutting tool machining are important ways of reducing production costs to ensure product quality and improve production efficiency. Researchers have paid much attention on these goals and explored a variety of effective processing methods for production practice [4][6][7].

The field technology, which includes the magnetic, electric, microwave and ultrasonic fields, among others, is an emerging technique in manufacturing research [4]. The field technology is often applied to the metal metallurgy and metal-modification treatment. The electric and magnetic treatment is a hot overall strengthening method compared with other strengthening treatment methods, such as heat treatment and coating. The study of the electromagnetic technology has a long history, but most studies focused on ferromagnetic material research and their application. However, not enough information is available regarding the electromagnetic coupling technology. The magnetization technology is a kind of microstructure improvement method for a ferromagnetic material, which is not realized by the temperature control. It strengthens not only the metal surface, but also the internal parts [8]. The magnetostriction behavior in a metal material occurs when metal is placed in an external magnetic field. It induces dislocation in a microstructure and increases the martensite content. The material microstructure becomes uniform, increasing the material microhardness [9]. The electric pulse treatment is another popular method for enhancing the tool performance using an external field. The atomic motion in a material can be increased when an electric field or a high current is applied. This reduces the energy required for activating a dislocation and accelerates the dislocation motion. Simultaneously, the dislocation entanglement is decreased, and the barrier of the slip system is overcome. Hence, the material shows higher plasticity and room temperature deformation ability [10].

The following common problems are faced by the tool-making industry: selection of a proper manufacturing process, including the external processing technology; research on the wear mechanism of the cutting tool, including tool wear analysis and monitoring; and the material removal mechanism, etc. Extensive and in-depth studies have been performed by researchers, but not many of these studies have focused on the processing mechanism and parametric influence of the external field technology for tool making. Jung et al. [10] found that pulse current can delay the growth and extension of a fatigue crack in aluminum alloy and enhance fatigue life by $55 \%$ compared with an untreated scanning electron microscopy fracture surface. Hosoi et al. [12] repaired cracks in stainless steel under electric field effect. An intense electric field was generated at the crack tip, which induced local high heat and thermal energy. A bridge was formed at the crack, which temporarily delayed the crack propagation. Padmakumar et al. [13] treated a milling blade with low-temperature and found that tool wear can be decreased by $10 \%$ at the flank and $27 \%$ at the knife nose, and less micro-cutting can be done at the tool edge. The tool magnetostrictive effect induced by the external magnetic field improved the uniformity in the material microstructure and enhanced its microhardness. Meanwhile, Yip and To et al. [14] found that the machined surfaces of titanium alloy can be improved by a magnetic field. The tool life can be prolonged by reducing the adhesive wear, flank wear, cutting tool edge, and vibration during machining. The cutting fluid damage to the environment was reduced effectively. Moreover, the magnetic field can promote oxidation. At the 
suitable cutting parameter, the generated oxide film on the cutting tool surface further inhibited the tool wear [15]. Ma et al. Error! Reference source not found. processed M42 high-speed steel cutting tools with pulse magnetization. Consequently, lattice distortion occurred, and a large number of dispersed carbides were precipitated in the matrix, which refined the microstructure [16]. Bataineh et al. [18] found that pulsed magnetic treatment can improve the wear resistance performance and service life of a twist drill. Electric current was generated in the magnetic field near the surface of the material and produced heat. The energy input is often associated with the local magnetic force near the surface. The magnetic force can change the microscopic structure material affecting the wear behavior and increase the dislocation production to prevent the crack generation. Dehghani et al. [19] conducted a machining experiment under a magnetic field. In their experiments, the magnetic field minimized the flank wear by as much as $94 \%$ and increased the cutting force by $66 \%$. Nadia et al. [20] found that the feed cutting, and radial forces were improved from $5 \%$ to $20 \%$ in a turning experiment when a direct magnetic field was applied.

Many researchers have studied the effect of the composite field on the metal material. Orgiu et al. [21] found a vacuumed electromagnetic environment can improve the material performance. Wang et al.[22] processed an unreformed TC11 alloy with a pulsed magnetic field, a pulse current, and a pulse electromagnetic alloy structure to explore their influences on structure, lattice parameter dislocation, and microhardness. After the processes, the content of the primary alpha phase in the alloy was increased, and the dislocation entanglement was decreased. Li et al. [23] studied the influence of an electromagnetic field on the aluminum alloy mechanical properties and microstructure. The pulse magnetic field can induce cyclical shocks in molten metal. Moreover, the eutectic silicon at the interface was distributed in block or short bar patterns, which benefited the interface bonding strength. Liu et.al [24] found that a pulsed magnetic field and a pulsed current can decrease the wear loss of the cemented carbides by nearly $35 \%$. The milling performance was significantly improved.

Previous literature indicated that pulse and magnetic field have a significant effect on the tool life. The electromagnetic field also has a positive effect on the tool. The influence of the TEMCP on the cutting process is studied herein. Further contributions to enrich the knowledge on the field technology are also made. The TEMCP is proven as an environment-friendly and sustainable manufacturing process with low costs. It reduces the waste of cutting tools and decreases the use of cutting fluid. Furthermore, the TEMCP is a green and efficient method for enhancing the tool life. The proposed method has strong applicability and research value.

\section{Theoretical background of the TEMCP and implementation method}

\subsection{TEMCP principle and equipment}

A self-development electromagnetic system is used to apply coupled electric and magnetic energy on the cutting tool. The whole system includes a field energy system (two pulse magnetic field generation systems with a pulse current system), a programmable logic controller (PLC) control system and a processing platform. The magnetic pulse generator is composed of a capacitor 
discharge system and an excitation coil. The magnetic field intensity can be changed by controlling the charging saturation of the capacitor bank. The magnetic pulse frequency is adjusted by changing the discharge beat of the capacitor bank. The multi-turn coil generates a magnetic field that fills the space. The equipment can produce a magnetic field with an intensity ranging from 0 to $3.5 \mathrm{~T}$ and a frequency from $1 \mathrm{~Hz}$ to $10 \mathrm{~Hz}$.

The pulse current excitation device is made up of a power supply system and a computer numerical control (CNC) controlled pulse system. The power system converts the alternating current at $380 \mathrm{~V}(50 \mathrm{~Hz})$ to a direct current with a maximum current of 5000A and a voltage ranging from 0 $\mathrm{V}$ to $10 \mathrm{~V}$. The pulsing system is composed of the PLC and a high-voltage switch. The impulse waveform and output direction are controlled by programming.

For the experiment implementation, the external alternating current is first pre-processed by the $\mathrm{CNC}$ power system. The treated current is then turned into pulse current with low voltage and high current to process the cutting tool with a pulsed electric field. At the same time, the capacitor system pre-processes the alternating current and charges the capacitors. A high-intensity pulse magnetic field is produced by discharging the capacitors, which magnetizes the tool. The whole process is finished in $1 \mathrm{~min}$.

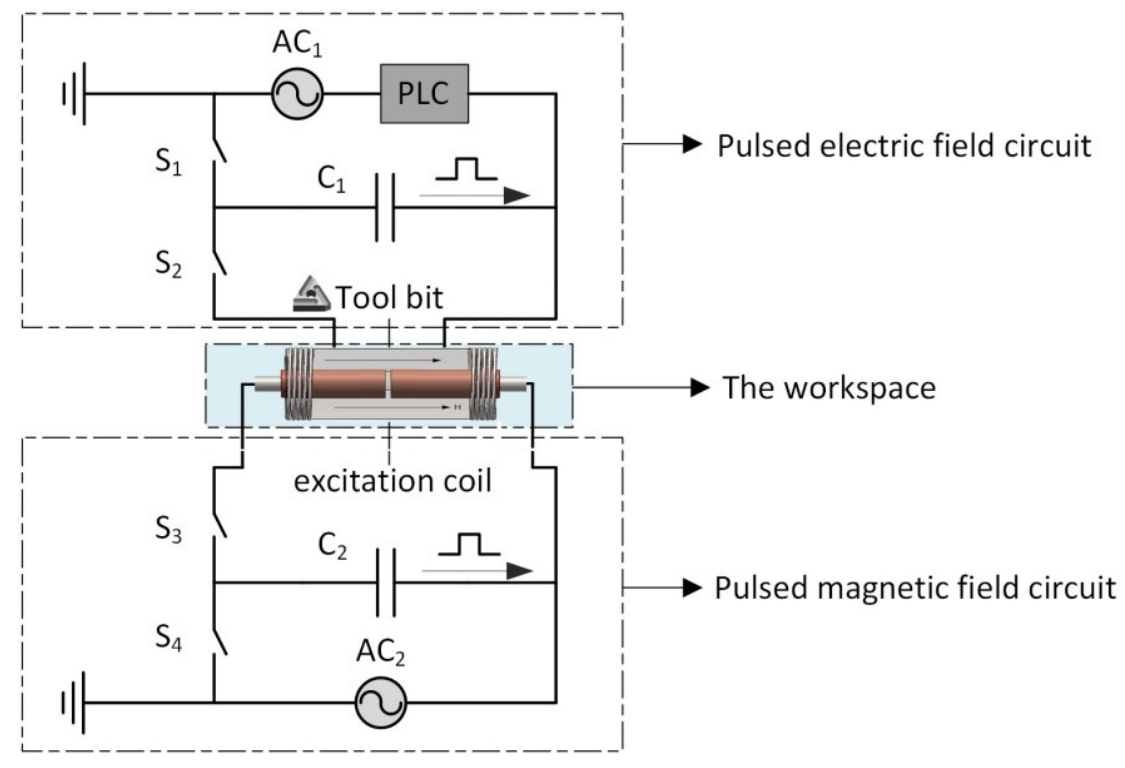

Figure 1. Schematic diagram of TEMCP

\subsection{Processing tool scheme using TEMCP}

In this study, seven trials were performed at the same cutting conditions. The untreated tool was used as a control group, while the other seven tools were treated with different TEMCP processing parameters (Table 1). The P10 cutting tool was made of tungsten-cobalt-titanium cemented carbide with high wear resistance and hardness. The main materials are tungsten carbide, cobalt, and titanium carbide, which are suitable for semi-finishing and finish machining for materials with high machining hardness. The surface quality request for these machining processes is high, which has an important research value. 


\begin{tabular}{|c|c|c|c|c|c|c|c|c|}
\hline Treatment $\quad$ Tool number & Tool 1 & Tool 2 & Tool 3 & Tool4 & Tool 5 & Tool 6 & Tool 7 & Tool 8 \\
\hline Magnetic intensity(T) & 0 & 0.5 & 0.5 & 0.5 & 1.0 & 1.5 & 1.5 & 2 \\
\hline Electric density $(\mathrm{A} / \mathrm{mm} 2)$ & 0 & 0.4 & 1.2 & 2.0 & 1.2 & 1.2 & 2.0 & 1.2 \\
\hline
\end{tabular}

\subsection{Experiment procedure}

The experiments were conducted on a G-CNC6135 lathe equipped with a P10 turning tool with a model number of 31303C. The workpiece was made of 45 quenched and tempered steel with $\Phi$ $100 \mathrm{~mm}$ diameter and $200 \mathrm{~mm}$ length. The mechanical property and hardness of the workpiece were enhanced after quenching and high-temperature tempering. A Kistler 9257B three-phase dynamometer and a Kistler 5019A multi-channel charge amplifier were used to monitor the cutting force. The signal was then converted by an A/D converter with a sampling frequency of $2 \mathrm{kHz}$. The tool wear was inspected by the VMS3020 image measuring instrument. Figure 2 illustrates the experimental equipment. All cutting parameters were kept the same to investigate the influence of the TEMCP on the tool life. The specific cutting parameters are as follows: $560 \mathrm{r} / \mathrm{min}$ spindle speed; $60 \mathrm{~mm} / \mathrm{min}$ feed rates; and $1 \mathrm{~mm}$ cutting depth. The sampling length of cutting was set to $95 \mathrm{~mm}$. After every test, the cutter blade was unloaded to measure the wear volume and the wear surface image under VMS3020 until tool failure occurred.

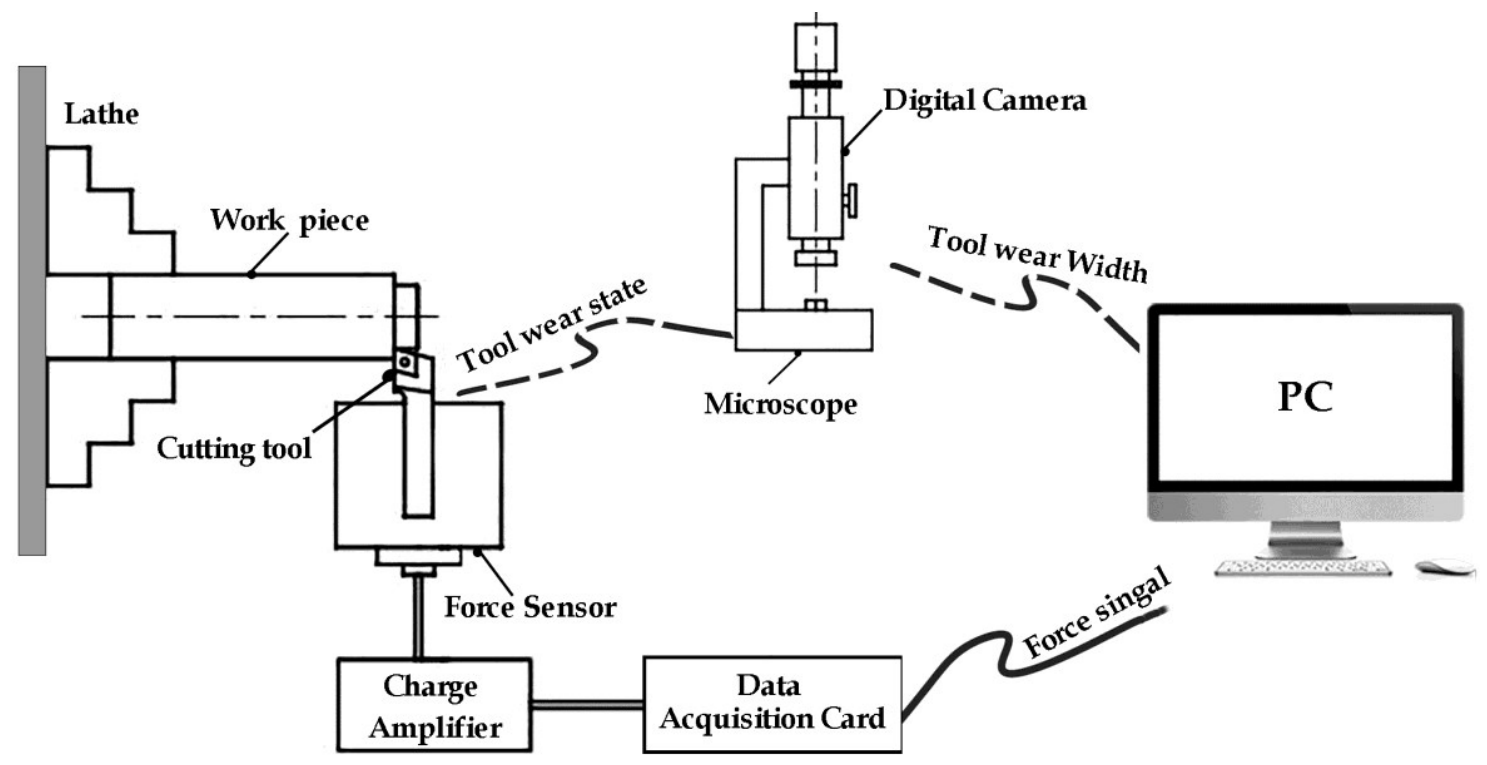

Figure 2. Experimental setup

\section{Experiment results}

\subsection{Selection of indicator and criteria for tool wear}

The performance change of the cutting tool affected by the TEMCP technique in the machining process must be determined accurately by the relationships of the tool characteristics. Macromechanics emerges accompanied with the machining process affects the state of the tool wear. The mechanical signal collection is simple, feasible and reliable. The relationship between the 
intensity of electromagnetic and mechanical properties must be studied to reveal the mechanism between the TEMCP and the tool life.

Blunt standard refers to the degree of wear, in which the tool cannot be used anymore. The tool wear mainly consists of various stages of flank wear (VB); hence, the ISO 08688-1 standard has defined $0.3 \mathrm{~mm}$ of VB as the tool failure limit. The cutting tool fails when the VB value of the tool reaches $0.3 \mathrm{~mm}$ and needs to be replaced. Figure 3 presents images of the cutting tool (Tool 1) wear at four stages: initial worn, normal worn, acutely worn, and tool failure.

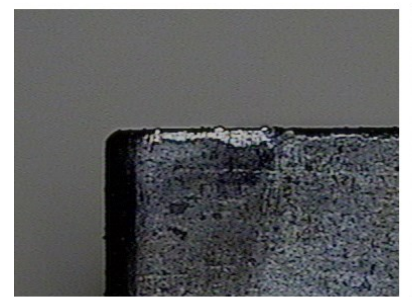

Initial worn

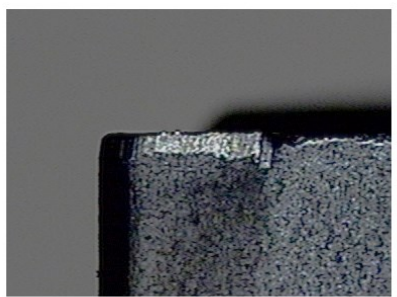

Normal worn

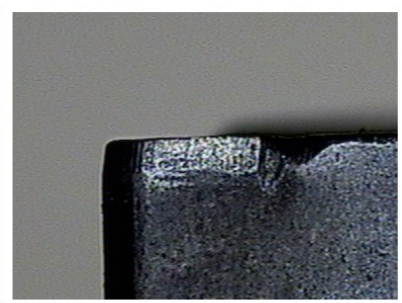

Acutely worn

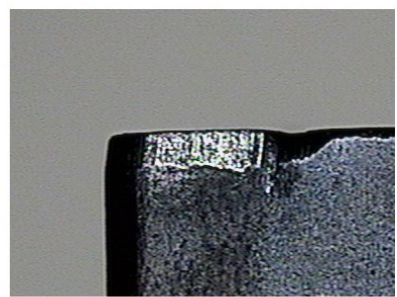

Tool failure

Figure 3. Images of the four different states representative of progressive tool wear

\subsection{Influence of the TEMCP on the tool life}

Figure 4 presents the tool wear curves of the eight tools. An untreated tool was used for four times, and the most stable cutting data were adopted to guarantee the accuracy of the control group. Consequently, first, the tool wear rose fast in the early state, then slowed down. The wear rate increased again when the tool was close to its failure limit. All wear curves conformed to the law of tool wear. Second, different TEMCP parameters enhanced the tool life to different degrees. Third, the tool life was most significantly improved when the magnetic field intensity was larger than $1.5 \mathrm{~T}$ or when the electric field intensity was $1.2 \mathrm{~V}$. The tool life was increased by 1.92 times when the magnetic field intensity was $1.5 \mathrm{~T}$ and the electric field intensity was $1.2 \mathrm{~V}$.

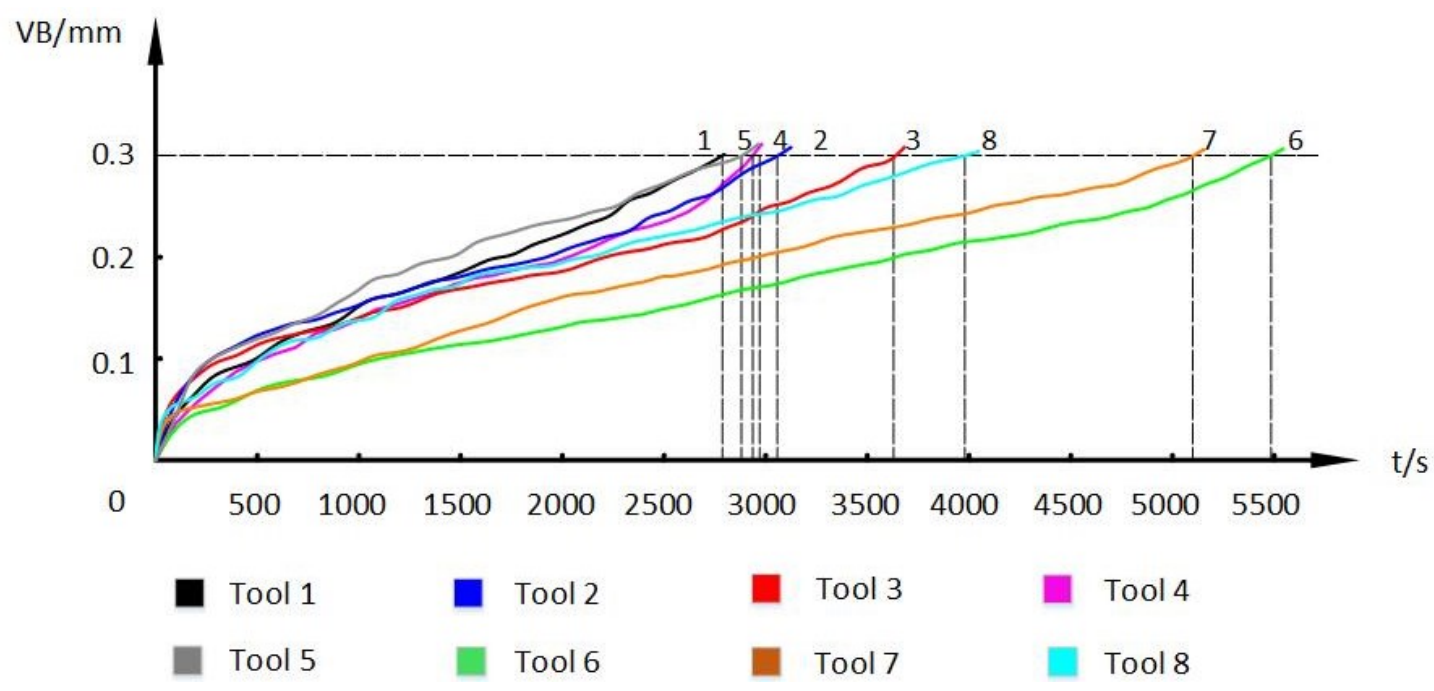

Figure 4. Experimental tool wear process curves for eight tools.

Figure 5 presents the specific TEMCP treatment parameters and their corresponding tool lives. Three kinds of electric field intensity and four kinds of magnetic field intensity were selected for the 
TEMCP treatment. Tool 1 presents an untreated with a tool life of $48.09 \mathrm{~min}$. The rest of the tool number and lives were marked in the figure. The tool life of P10 was increased by $92.07 \%$ compared with that of Tool 1. As a result, first, no obvious change in the tool life between the treated tool and the UT was observed when the magnetic field intensity was smaller than $1 \mathrm{~T}$, but it was significantly improved when the magnetic field intensity was increased to $1.5 \mathrm{~T}$. Second, the tool life was significantly changed as the magnetic field increased when the electric field intensity was $1.2 \mathrm{~V}$. The lifetime reached $67.42 \mathrm{~min}$ when the magnetic field strength was $1.5 \mathrm{~T}$. The tool life can be increased to 1.87 times under the same electric field, but can only be increased to 1.24 times under the same magnetic field intensity $(0.5 \mathrm{~T})$. Thus, we preliminarily judge that the magnetic field plays a leading role in the effect of the TEMCP treatment on the tool life; however, further exploration is needed.

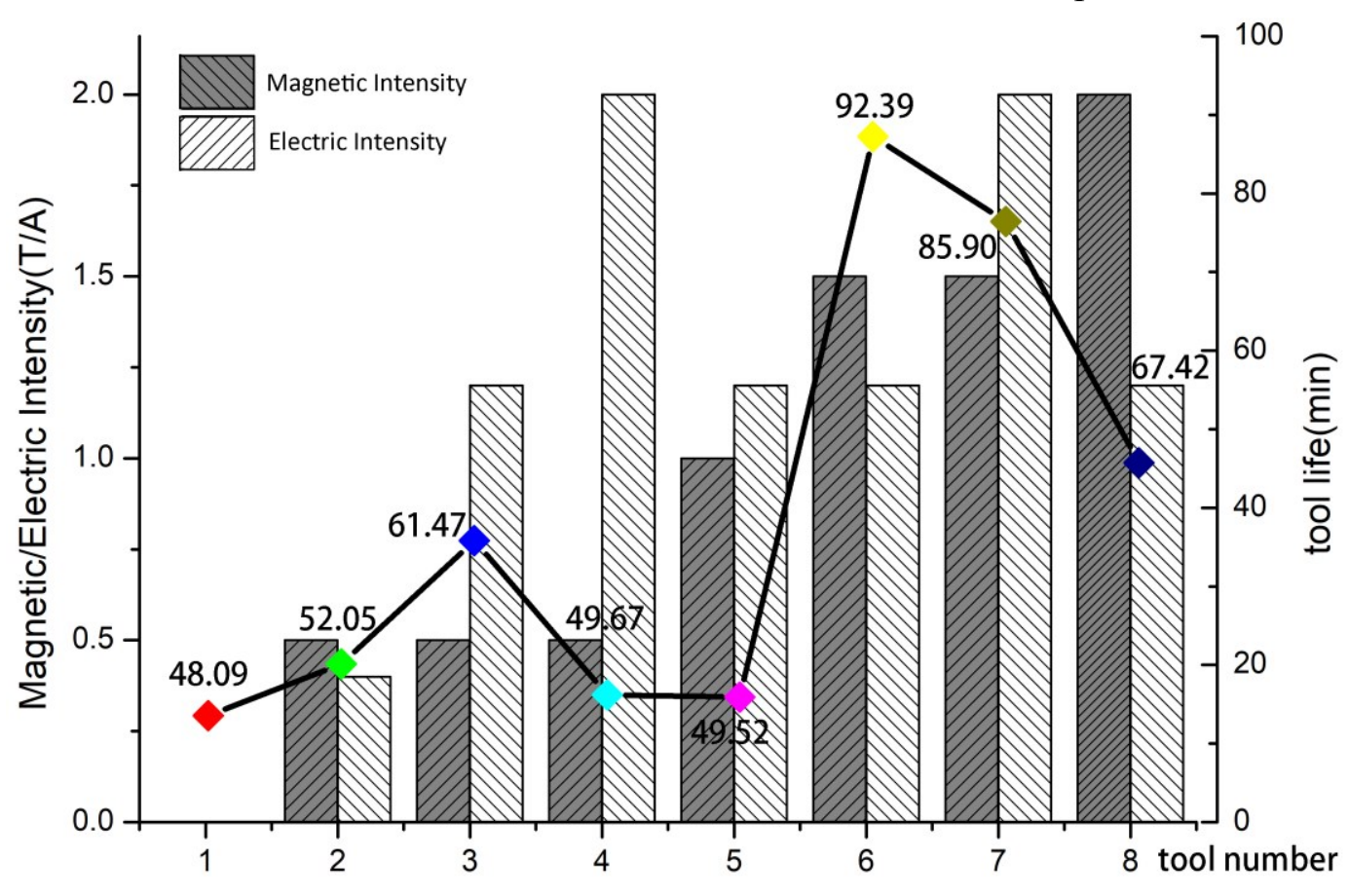

Figure 5. TEMCP treatment parameters corresponding to the tool wear time

The VB of the eight tools was recorded at the same time and the images were taken as shown in Figure 6. A machining duration of approximately $3000 \mathrm{~s}$ was selected because the Tool 1 failed at this time. The wear images were more intuitive and representative. When Tool 1 failed, the wear degree of the other cutting tools was less than that of Tool 1 . Tools 2, 4, and 5 were on the verge of failure, while Tools 6 and 7 were still in the normal wear stage. Tools 3 and 8 were in the severe wear stage. The TEMCP greatly influenced the wear degree of the back-cutting surface. 


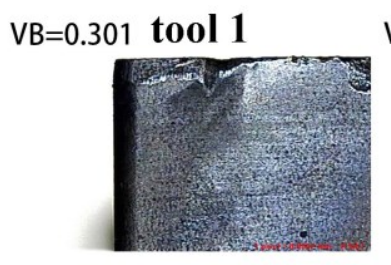

$\mathrm{VB}=0.299$ tool 5

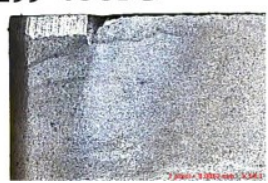

$\mathrm{VB}=0.287$ tool 2

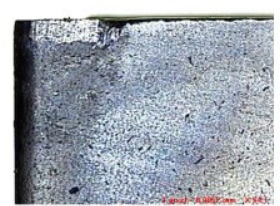

$V B=0.168$ tool 6

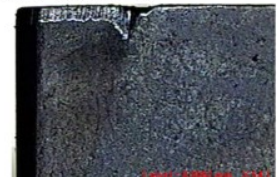

$\mathrm{VB}=0.237$ tool 3

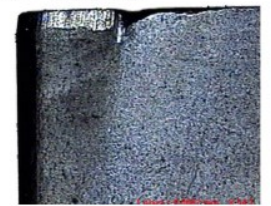

$\mathrm{VB}=0.198$ tool 7

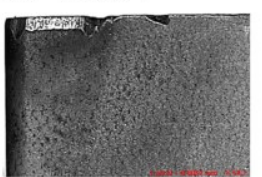

$V B=0.291$ tool 4

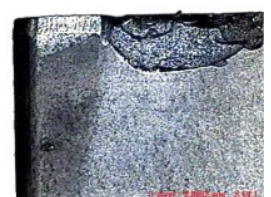

$V B=0.238$ tool 8

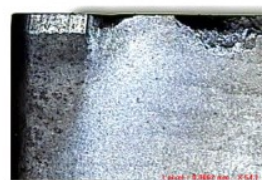

Figure 6. VB wear of different cutting tools at the same time

\subsection{Influence of the TEMCP on the maximum cutting force}

The cutting signal of a tool is an indicator of the running state of the cutter. The maximum value of the cutting force has a close relationship with the tool wear and increases with the increase of the cutting time. Figure 7 shows the comparison results of the maximum values of the cutting force of the eight cutters. The maximum value of Tool 1 rapidly increased with the cutting distance, while that the maximum value of the cutting force of the seven processed tools decreased by $56 \%$ that of Tool 1. TEMCP treatment can improve the cutting stability; hence the TEMCP had a significant effect on the maximum value of the cutting force.

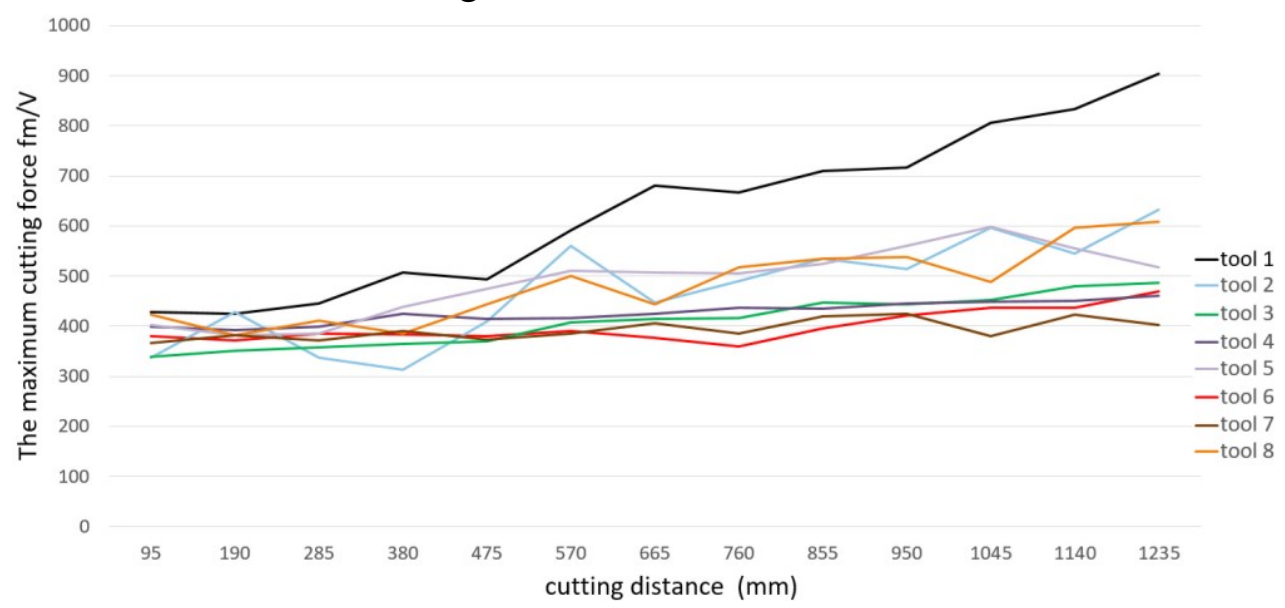

Figure 7. TEMCP effects on the maximum value of the cutting force

\subsection{Comparison of the machining vibration on the Fast Fourier transform analysis}

Fast Fourier transform (FFT) of the cutting force could reflect the machining vibration of the turning system generated in the TEMCP. The force signal of Tool 1 (first line) and Tool 6 (second line) from the time domain was converted to a frequency domain (Figure 8) at five cutting distances. The sample frequency was $10 \mathrm{~Hz}$. The $\mathrm{X}$ axis in the graph represents the corresponding frequency, while the $\mathrm{Y}$ axis represents the cutting force amplitude. Figure 8 presents a comparison between the peak values of both samples. All peak amplitudes of the TEMCP in FFT were lower than that of the untreated tool for a cutting distance of $380-2850 \mathrm{~mm}$. The kinetic energy of the vibration in the manufacturing system would be converted to heat energy; hence, the overall turning vibration was 
suppressed by the TEMCP. The tool wears increased with the cutting distance increase; therefore, the vibration of both tools working at the same condition as the cutting distance increased compared to that in Figure 8. With an increasing cutting distance, the vibration amplitudes of Tool 6 were lower than those of Tool 1 all the time until Tool 1 failed. The maximum peak amplitude in FFT of the TEMCP was only $14.09 \mathrm{~N}$, especially at the cutting distance of $1805-1900 \mathrm{~mm}$, the maximum peak amplitude in FFT of TEMCP was only $14.09 \mathrm{~N}$ while the value of Tool 1 was $54.07 \mathrm{~N}$. Referring to FFT of both samples at all cutting distances, the waveform of Tool 6 was flatter than that of Tool 1. In addition, the peak value was much lower than that of Tool 1. These results explain that the tool wear was remarkably reduced, and the tool life was enhanced under the TEMCP treatment.

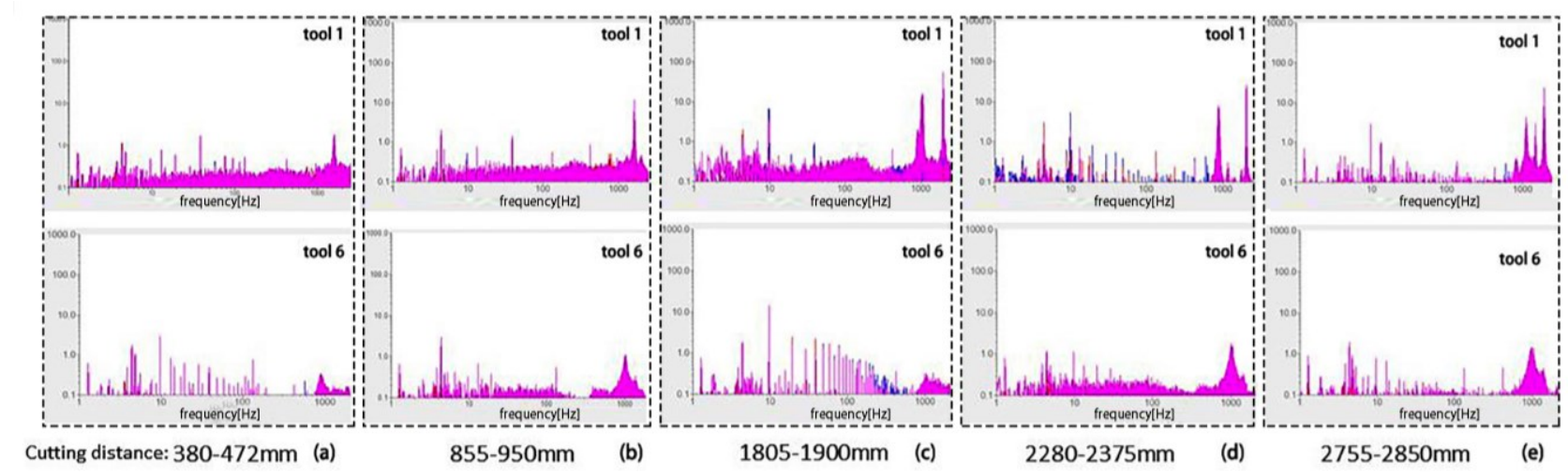

Figure 8 . FFT of the feed force generated by the TEMCP at the cutting distances of (a) $380-472 \mathrm{~mm}$, (b) $855-950 \mathrm{~mm}$, (c) $1805-1900 \mathrm{~mm}$, (d) $2280-2375 \mathrm{~mm}$, and (e) $2755-2850 \mathrm{~mm}$

\subsection{Simple correlation analysis of the tool life index and TEMCP parameters}

A descriptive statistical analysis of the tool life index and the electromagnetic field parameters was performed as depicted in Table 2. The normal distribution probabilities of the tool life, wear cycles, VB, magnetic intensity and electric density were all larger than 0.05 , which is consistent with those in normal Q-Q diagram in SPSS. The distribution met the basic requirements of correlation analysis and statistics. Meanwhile, the frequency of the magnetic field and electric field did not participate in the later analysis because they did not meet the normal distribution.

Table 2. TEMCP process parameters and tool life index

\begin{tabular}{lllllll}
\hline Items & Mean value & standard deviation & peak & skewness & Kolmogorov-Smirnov & Normal probability distribution \\
\hline Tool life(min) & 63.6375 & 17.06246 & 0.639 & 0.981 & 0.251 & 0.145 \\
Wear cycles (times) & 38.88 & 10.148 & 0.638 & 0.964 & 0.251 & 0.147 \\
VB(mm) & 0.30775 & 0.003196 & 1.446 & 0.363 & 0.220 & 0.200 \\
Magnetic intensity(T) & 0.938 & 0.6781 & 1.078 & 0.294 & 0.241 & 0.193 \\
Magnetic frequency(Hz) & 0.088 & 0.0354 & 8.000 & 2.828 & 0.513 & 0.000 \\
Electric density $(\mathrm{V})$ & 1.150 & 0.6908 & 0.217 & 0.413 & 0.279 & 0.067 \\
Electric frequency(Hz) & 43.75 & 17.678 & 8.000 & 2.828 & 0.513 & 0.000 \\
\hline
\end{tabular}

Table 2 shows that the tool life and the wear times were moderately correlated with the 
magnetic field strength, while the VB was negatively correlated with the magnetic field strength; and the tool life, wear times, and VB had a low correlation with the electric field intensity. The magnetic intensity and tool wear indicators are generally more relevant. The stronger the magnetic intensity, the lower the degree of the tool surface wear. The tool life and the wear times were higher, which is consistent with the actual experimental results, indicating that the magnetic intensity is the leading factor affecting the tool life.

\begin{tabular}{l} 
Table 3. Simple correlation analysis of the tool life index and TEMCP \\
\cline { 2 - 3 } \begin{tabular}{lll} 
Items & Magnetic intensity & Electric density \\
\hline Tool life & 0.693 & 0.381 \\
Wear cycles & 0.684 & 0.390 \\
VB & $-0.799^{*}$ & 0.188 \\
\hline
\end{tabular}
\end{tabular}

The abovementioned experimental results suggest that the TEMCP can change the cutting mechanisms, including the tool life, cutting force, and machining vibration. The magnetic field is the main factor. The same batch of cutting tools was treated at the same position in the self-development electromagnetic system to ensure the reliability of the experiments. The workpieces were then machined under the same cutting conditions. Tool 6 was tested thrice under the same conditions to ensure repeatability. The tool life of Tool 6 was approximately two times of that of Tool 1.

\section{Discussion of TEMCP mechanism}

The reasons why the TEMCP field improved the tool life are as follows:

1) Electro-plastic effect: the electro-plastic effect is a comprehensive effect combined with many physical effects, including the Joule effect, magnetic compression effect, pure electro-plastic effect, skin effect, and thermo-electric effect. The Joule effect plays a leading role in the electro-plastic effects produced by different current densities. The temperature rise caused by the Joule thermal effect is expressed as:

$$
\Delta T=\rho J^{2} t_{p} /\left(c_{p} d\right),(1)
$$

where $\rho$ is the material resistance; $J$ is the current density; $t_{p}$ is the pulse duration time; $c_{p}$ is the heat capacity of constant pressure; and $d$ is the material density. The electro-plastic effect can increase the dislocation value and the dislocation movement and creep, decrease the flow stress, and improve the material plasticity. Song et al. [25] found that electropulsing can refine the microstructure of Ti-22Al-27NB sheets with an elongation that reaches $19.4 \%$. The electropulsing treatment is an effective method for improving the titanium alloy plasticity.

2) Magnetostriction effect: the grain of a cutter material is refined at an external magnetic field, which improves spring back of the material in the removal process. The thermodynamic equation of the magnetization effect is shown in Eq. (2) as follows: 


$$
T d S=C_{m} d T-u_{0} H d M+p d V
$$

where, $C_{m}$ is the magnetization heat capacity, which is a positive number, $u_{0}$ is the permeability; $H$ is the magnetic field intensity; $M$ is the magnetization intensity; $p$ is the internal stress that can be considered similar to the size of the external stress in the material deformation process; and $V$ is the volume of the deformed material. The temperature is increased in the magnetization process, $d T>0$, under the electric field effect. The magnetization is finished before the turning processing, and no external stress exists, $p d V=0$. Equation (1) becomes $T d S=C_{m} d T-u_{0} H d M$, $d T=\rho J^{2} t_{p} /\left(c_{p} d\right) . C_{m}, d T, u_{0}, H$, and $d M$ are $>0$. The value of the $T d S$ is unknown. When $T d S>0$, the system gives off heat. In contrast, when $T d S<0$, the system takes in heat. In the process of adiabatic demagnetization, $T d S=0$. Equation (2) becomes $d T=\frac{u_{0} H d M-p d V}{C_{m}}$. External work is done to the material because $d M<0, C_{m}>0$. Plastic deformation also occurs in the material processing, $p d V>0$; hence, the system temperature decreases when $d T<0$. Therefore, it is very important to study the coupling law of the three energy fields (thermal-TEMCP -deformation) in electromagnetic coupling. Cai et al. [26] found that the product of the magnetostriction amplitude and the stress magnitude is nearly a constant during the magnetic field treatment. The removal of the residual stress in metal can improve the mechanical properties.

3) Residual stress removal: residual stress in a workpiece is caused by non-uniform stress field, strain field, temperature field, and non-uniform structure. The existence of the residual stress directly affects the workpiece machining accuracy, fatigue strength, and size stability and the tool life. Based on elecro-dislocation dynamics and plastic micromechanics, both electric and magnetic fields can increase the internal dislocation density, make the dislocation distribution uniform, and reduce the residual stress. A sufficiently large current energy will generate sufficient acting stress on the dislocation structure and open up the dislocation pileup to make it slip. The crystal will be subjected to plastic deformation, and the residual stress will be reduced or eliminated. A more concentrated electropulse will have a more significant effect on the removal rate of the residual stress. The magnetic field can encourage material dislocation. Affected by an external magnetic field, the crystal will slip; the workpiece will produce plastic deformation; the stress will be released; and the residual stress will be reduced. Barney [27] measured the extent of residual stress reduction caused by a particular pulsed magnetic treatment. Residual stress reductions of 4-7\% were obtained for that particular pulsed magnetic treatment used on lower initial stress-level specimens. Meanwhile, stress reductions of $8-13 \%$ were obtained higher initial stress levels. 


\section{Conclusions}

The field technology is a clean and sustainable metal-modification technology that can improve the performance of the surface of whole materials. It also helps reduce usage of the cutting fluid and environmental pollution in machining difficult-to-machine materials. This study introduced a new field technology. The P10 tool strengthened in the TEMCP was used to turn the No. 45 quenched and tempered steel bar to observe the effect of the TEMCP on a carbide cutter. The experimental results showed that the cemented carbide tool processed by the TEMCP has a longer life in dry machining, which can greatly reduce the tool replacement frequency. The TEMCP can improve the tool life and reduce the maximum tool force. This research obtained a breakthrough result that enriches the field technology theory and fills the knowledge gap in the TEMCP treatment of carbide turning tools. The TEMCP has an abundant research value and a broad application prospect. The performed experiment adopted a self-designed electromagnetic coupling system, which is green and efficient in the treatment process and beneficial to the environment and sustainable development. The following conclusions are were obtained in this study:

1. The tool life was improved to varying degrees under the TEMCP treatment. Different TEMCP parameters had different influences on the tool life.

2. The maximum of the cutting force was sharply decreased by $56 \%$ after the TEMCP treatment, thereby prolonging the cutting stroke and vibration and deferring the tool wear process.

3. The effects of the tool life index (i.e., tool life, wear cycles, and VB) and TEMCP parameters (i.e., magnetic intensity and electric density) were statistically analyzed herein. Magnetic intensity was found to be the dominant factor affecting the tool life, which is consistent with the experimental results.

4. The tool life in the electromagnetic field increased because of three main reasons: electro-plastic effect; magnetostriction effect; and residual stress relief. The theory of thermal - TEMCP - deformation coupling theory must be further analyzed.

The TEMCP can significantly improve the service life of cemented carbide tools, reduce the cutting wear, and contribute to sustainable manufacturing and environmental protection. The experimental results showed that the magnetic field is the dominant factor for enhancing the tool life. The specific reasons for this must be further studied. In conclusion, the TEMCP is a sustainable, environment-friendly, and efficient field technology with broad research value.

\section{Funding information}

The authors wish to acknowledge the financial support by the National Nature Science Foundation of China (no.51705348), Sichuan science and technology program (no.2019YFG0359) and the Research Funds for the Central University (no.2019SCUH0013).

\section{Reference}


[1]. Wang M, Wang J (2012) CHMM for tool condition monitoring and remaining useful life prediction. Int J Adv Manuf Technol 59(5-8):463-471. https://doi.org/ doi: 10.1007/s00170-011-3536-7

[2]. Liao XP, Zhou G, Zhang ZK, Lu J, Ma JY (2019) Tool wear state recognition based on GWO SVM with feature selection of genetic algorithm. Int J Adv Manuf Technol 104:1051-1063 . https://doi.org/10.1007/s00170-019-03906-9

[3]. Chen XZ, Li CB, Jin Y, Li L (2018) Optimization of cutting parameters with a sustainable consideration of electrical energy and embodied energy of materials. Int J Adv Manuf Technol 96:775-788. https://doi.org/10.1007/s00170-018-1647-0

[4]. Krolczyk GM, Nieslony P, Legutko S (2015) Determination of tool life and research wear during duplex stainless steel turning. Archives of Civil \& Mechanical Engineering 15(2): 347-354. https://doi.org/ doi: 10.1016/j.acme.2014.05.001

[5]. Sttkı A, Hasan G, İlyas U (2015) A review of cryogenic treatment on cutting tools. Int J Adv Manuf Technol 78:1609-1627. https://doi.org/ doi: 10.1007/s00170-014-6755-x

[6]. Liu SY; Shin YC (2019) Additive manufacturing of Ti6Al4V alloy: A review. Materials \& design 164: 107552. https://doi.org/ doi: 10.1016/j.matdes.2018.107552

[7]. Zhang JH, Liu JX (2014) Heat treatment process improvement of M42 high speed steel. Heat Treatment of Metals 39(11):132-134. https://doi.org/ doi: 10.13251/j.issn.0254-6051.2014.11.033

[8]. Fu YM, Wang SX, Huo LJ, Chen GX (2013) Experimental study for strong alternating magnetic treatment of diamond segment. Journal of Plasticity Engineering 20(5):126-129. https://doi.org/ doi: 10.3969/j.issn.1007-2012.2013.05.025

[9]. Zhao WX, Yao HM, Liang ZQ, Ma LP, Wang XB, Zhou TF (2014) Effects of Pulsed Magnetic Field on the Micro-Hardness of HSS Cutting Tool Materials. Transactions of Beijing Institute of Technology 34(7): 661-665. https://doi.org/ doi: 10.15918/j.tbit1001-0645.2014.07.006

[10]. Xu XL, Zheng GG, Wang HD, Wang T (2017) Research progress on the application of electro-plastic effect in materials processing. Journal of Plasticity Engineering 24(6):1-7. https://doi.org/ doi: 10. 3969 /j. issn. 1007-2012. 2017.06. 001

[11]. Jung J, Ju Y, Morita Y, Toku Y (2016) Effect of pulsed electric current on fatigue crack in Al. Procedia Structural Integrity. 2:2989-2993. https://doi.org/ doi: 10.1016/j.prostr.2016.06.374

[12]. Hosoi A, Nagahama T, Ju Y (2015) Fatigue crack healing by a controlled high density electric current field. Materials Science \& Engineering: A (Structural Materials: Properties, Microstructure and Processing) 533:38-42. https://doi.org/ doi: 10.1016/j.msea.2011.11.024

[13]. Padmakumar M, Dinakaran D, Ravikumar S G, \& Vijay, S. K. S (2015) Performance Evaluation of Cryogenically Treated Tungsten Carbide Insert on Face Milling of Grey Cast Iron. Applied Mechanics and Materials 6:813-814. https://doi.org/ doi: 10.4028/www.scientific.net/AMM.813-814.569

[14]. Yip, WS, To, S (2017) Tool life enhancement in dry diamond turning of titanium alloys using an eddy current damping and a magnetic field for sustainable manufacturing. Journal of Cleaner Production 168: 929-939. https://doi.org/ doi: 10.1016/j.jclepro.2017.09.100

[15]. Zhu T, Liu Z, Shen JB (2015) Influence of magnetic treatment on wear resistance of W6Mo5Cr4V2 high speed steel. Heat Treatment of Metals 40(10):145-151. https://doi.org/ doi: 10.13228/j.boyuan.issn1001-0963.20140443

[16]. Ma L, Wang X, Liang Z, Liu Y, \& Zhang D (2019) Machining mechanism in pulsed 
magnetic treatment for micro-tools. Int J Adv Manuf Technol 101:2391-2406. https://doi.org/ doi: $10.1007 / \mathrm{s} 00170-018-3105-4$

[17]. Ma LP, Liang ZQ, Wang XB, Zhao, WX, Zhou, TF, \& Yao, HM (2013) Effect of Low-Frequency Pulsed Magnetic Treatment on Micro-Hardness of High Speed Steel. Advanced Materials Research 797:663-666. https://doi.org/ doi: 10.4028/www.scientific.net/AMR.797.663

[18]. Bataineh O, Klamecki B, Koepke B G (2003) Effect of pulsed magnetic treatment on drill wear. Journal of Materials Processing Tech 134(2):190-196. https://doi.org/ doi: 10.1016/S0924-0136(02)01002-6

[19]. Dehghani A, Amnieh, SK, Tehrani, AF, \& Mohammadi, A (2017) Effects of magnetic assistance on improving tool wear resistance and cutting mechanisms during steel turning. Wear. 384-385:1-7. https://doi.org/ doi: 10.1016/j.wear.2017.04.023

[20]. Nadia, JN, Aaron, F, \& Azuddin, M (2017) Influence of electromagnetic field on metal cutting in turning operation of AISI 1018 low carbon steel. Materials Science \& Engineering Conference Series. Materials Science and Engineering Conference Series. https://doi.org/ doi: 10.1088/1757-899X/210/1/012066

[21]. Orgiu E, George J, Hutchison J. A, Devaux E, Dayen J. F, Doudin, B, Stellacci F, Genet C, Schachenmayer J, Genes C, Pupillo G, Samorì P, Ebbesen TW (2015) Conductivity in organic semiconductors hybridized with the vacuum field. Nature Materials 14(11):1123-1129. https://doi.org/ doi: 10.1038/NMAT4392

[22]. Wang L, Liu J, Yang Y, Yang G, Wei C, Wang LB, Gao Y (2018) Effects of electromagnetic treatment on microstructures and properties of TC11 titanium alloy. The Chinese Journal of Nonferrous Metals 28(5):931-937. https://doi.org/ doi: 10.19476/j.ysxb.1004.0609.2018.05.09.(in Chinese)

[23]. Li GR, Xue F, Wang HM, Zheng R, Zhu Y, Chu QZ, Cheng JF (2016) Tensile properties and microstructure of 2024 aluminum alloy subjected to the high magnetic field and external stress. Chinese Physics B 25(10): 106201. https://doi.org/ doi: 10.1088/1674-1056/25/10/106201

[24]. Liu J, Wei C, Yang G, Wang, LB,Wang L, Wu XL, Jiang K, Yang Y (2018) A Novel Combined Electromagnetic Treatment on Cemented Carbides for Improved Milling and Mechanical Performances. Metallurgical \& Materials Transactions A:1-11. https://doi.org/ doi: 10.1007/s11661-018-4740-y

[25]. Song H, Wang ZJ, He XD (2013) Improving in plasticity of orthorhombic Ti2AlNb-based alloys sheet by high density electropulsing. Trans. Nonferrous Met. Soc. China 23: 32-37. https://doi.org/ doi:10.1016/S1003-6326(13)62425-5

[26]. Cai ZP, Duan XJ, Lin J, Zhao HY (2011) Magnetostriction varieties and stress relief caused by pulsed magnetic fi eld. Front. Mech. Eng, 6(3): 354 - 358. https://doi.org/ doi: 10.1007/s11465-011-0123-4

[27]. Barney EKlamecki (2003) Residual stress reduction by pulsed magnetic treatment. Journal of Materials Processing $\quad$ Technology 141(3): 385-394. https://doi.org/10.1016/S0924-0136(03)00387-X 